\title{
Foreword
}

\section{Cerebellopontine Angle Lesions (Part 2)}

\author{
James K. Liu ${ }^{1,2}$ \\ ${ }^{1}$ Department of Neurological Surgery, Rutgers, Neurological Institute \\ of New Jersey, Rutgers University-New Jersey Medical School, \\ Newark, New Jersey, United States \\ 2 Department of Otolaryngology-Head and Neck Surgery, Rutgers, \\ Neurological Institute of New Jersey, Rutgers University-New Jersey, \\ Medical School, Newark, New Jersey, United States
}

J Neurol Surg B 2019;80(suppl S3):S265-S266.

We are proud to present the second installment of Cerebellopontine Angle Lesions (Part 2). This collection of operative videos covers a broad spectrum of surgical approaches for a variety of cerebellopontine angle (CPA) lesions. We begin with a compendium of videos demonstrating the technique for removal of vestibular schwannomas of various sizes, including the retrosigmoid, translabyrinthine, and middle fossa approaches. The more novel, yet controversial, endoscopic transcanal transpromontorial approach is also highlighted. Next, we showcase the surgical strategies for meningiomas of the CPA. Although most of these tumors can be removed via the standard retrosigmoid approach, more complex petrous and petroclival meningiomas may require a more complex surgical approach (anterior petrosectomy or combined petrosal). The surgical treatment of neurovascular compression syndromes including trigeminal neuralgia, hemifacial spasm, and

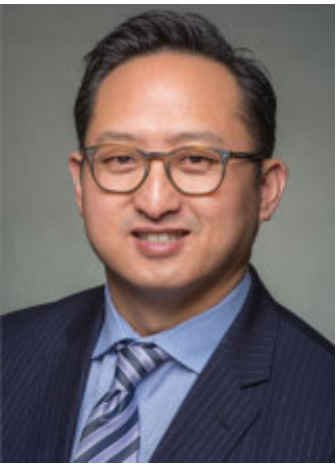

James K. Liu, MD geniculate neuralgia are also demonstrated. Lastly, the operative management of CPA epidermoid tumors and treatment of vascular lesions, such as dural arteriovenous fistulas and dissecting aneurysms, are exhibited. Appropriate approach selection and meticulous surgical technique with careful handling of neurovascular structures are paramount for achieving a maximal extent of resection while avoiding cranial nerve injury and vascular compromise. In this issue of Skull Base: Operative Videos, of Journal of Neurological Surgery: Part $B$, we showcase a library of video manuscripts to highlight the variety of important surgical techniques required for one to venture into the CPA. Please visit our YouTube channel, "Skull Base: Operative Videos," at www. thieme.com/skullbasevideos, to view the complete collection of operative videos.

Address for correspondence James K. Liu, MD, Department of Neurological Surgery, Center for Skull Base and Pituitary Surgery, Rutgers Neurological Institute of New Jersey, Rutgers University, New Jersey Medical School, 90 Bergen Street, Suite 8100 Newark, NJ, United States (e-mail: james.liu.md@rutgers.edu).
DOI https://doi.org/

10.1055/s-0039-1688825. ISSN 2193-6331. (c) 2019 Georg Thieme Verlag KG Stuttgart - New York

\section{License terms}

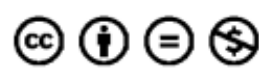

